\title{
An hamiltonian from the Dirac Equation and Two Flavor Neutrino Oscillations
}

\author{
Christian Rakotonirina \\ rakotonirinachristianpierre@gmail.com \\ Institut Supérieur de Technologie d'Antananarivo, IST-T \\ Laboratoire de la Dynamique de l'Atmosphère, \\ du Climat et des Océans, DYACO, University of Antananarivo
}

March 10, 2018

\begin{abstract}
This work represents the expression, eigenvalues, eigenstates of an hamiltonian operator obtained from the Dirac equation and the application of the eigenstates of this hamiltonian for formulating the transition probability of two flavor neutrino oscillations.
\end{abstract}

Keywords : Kronecker product, Tensor product, Free spin- $\frac{1}{2}$ fermion, Positive energy, Negative energy, Transition probability.

\section{Introduction}

When we hear the word "hamiltonian" it comes to us that it is "energy", more precisely "operator energy". Knowing this operator for a system we can construct the evolution equation of the system. But as the title say it will be from an evolution equation which is the Dirac equation that an other hamiltonian will be extracted. It turns that we will have other evolution equation. This hamiltonian should be simpler than the Dirac hamiltonian. We can see in [1] how to obtain this hamiltonian from the Dirac equation.

The approximation of the $E=\sqrt{p^{2}+m^{2}} \approx p+\frac{m^{2}}{2 E}[5]$, of the energy of the Dirac theory has been used for getting the pontecorvo neutrino oscillation formula. Thus the results obtained by Pontecorvo are approximates. Later exact formula for neutrino oscillations has been obtained in [6]. Therefore 
for obtaining exact results an hamiltonian for the same energy will be a help. So, an hamiltonian extracted from the Dirac equation will be a candidate for replacing the approximation above, for obtaining exact formulae.

In the first section we will talk about the hamiltonian. In the second section, we will apply it for formulating the transition probability for two flavor neutrino oscillations.

\section{Energy in the Dirac theory}

The Dirac equation

$$
i \hbar \gamma^{\mu} \partial_{\mu} \psi-m c \psi=0
$$

is the quantum relativistic equation for a free spin- $\frac{1}{2}$ fermion, where the $\gamma^{\mu}$ 's are the gamma matrices. In this equation $\hbar$ is the Planck constant, $c$ the speed of light, $m$ the mass of the spin- $\frac{1}{2}$ fermion and $\psi$ is its wave function.

The gamma matrices may be written as tensor product or Kronecker product of the Pauli matrices

$\sigma^{1}=\left(\begin{array}{ll}0 & 1 \\ 1 & 0\end{array}\right), \sigma^{2}=\left(\begin{array}{cc}0 & -i \\ i & 0\end{array}\right), \sigma^{3}=\left(\begin{array}{cc}1 & 0 \\ 0 & -1\end{array}\right)$ and the $2 \times 2$-unit matrix $\sigma^{0}=\left(\begin{array}{ll}1 & 0 \\ 0 & 1\end{array}\right)$

$\gamma_{D}^{0}=\sigma^{3} \otimes \sigma^{0}, \gamma_{D}^{1}=i \sigma^{2} \otimes \sigma^{1}, \gamma_{D}^{2}=i \sigma^{2} \otimes \sigma^{2}, \gamma_{D}^{3}=i \sigma^{2} \otimes \sigma^{3}$ are the gamma matrices in the Dirac representation.

$\gamma_{W}^{0}=\sigma^{1} \otimes \sigma^{0}, \gamma_{W}^{1}=-i \sigma^{2} \otimes \sigma^{1}, \gamma_{W}^{2}=-i \sigma^{2} \otimes \sigma^{2}, \gamma_{W}^{3}=-i \sigma^{2} \otimes \sigma^{3}$ are the gamma matrices in the Weyl representation.

Writting the gamma matrices of this manner, then the resolution of the Dirac equation by using the Kronecker product of matrices gives rise to the following operator hamiltonians ,

$$
\begin{aligned}
& \hat{h}_{D}=\epsilon c p \sigma^{1}+m c^{2} \sigma^{3} \\
& \hat{h}_{W}=m c^{2} \sigma^{1}+\epsilon c p \sigma^{3}
\end{aligned}
$$

respectively in the Dirac and Weyl representations.

The eigenvalues of $\hat{h}_{D}$ are the positive energy $E=+\sqrt{c^{2} p^{2}+m^{2} c^{4}}$ and the negative energy $-\sqrt{c^{2} p^{2}+m^{2} c^{4}}$, associated respectively to the eigenvectors $\nu_{D}=\sqrt{\frac{E+m c^{2}}{2 E}}\left(\begin{array}{c}1 \\ \frac{\epsilon c p}{E+m c^{2}}\end{array}\right)$ and $\overline{\nu_{D}}=\sqrt{\frac{E+m c^{2}}{2 E}}\left(\begin{array}{c}-\frac{\epsilon c p}{E+m c^{2}} \\ 1\end{array}\right)$, with $\epsilon$ the sign of the spin. For $\hat{h}_{W}$ the eigenvectors associated to the same eigenvalues are 
$\nu_{W}=\frac{m c^{2}}{\sqrt{2 E(E-\epsilon c p)}}\left(\begin{array}{c}1 \\ \frac{E-\epsilon c p}{m c^{2}}\end{array}\right)$ and $\overline{\nu_{W}}=\frac{m c^{2}}{\sqrt{2 E(E+\epsilon c p)}}\left(\begin{array}{c}\frac{E+\epsilon c p}{m c^{2}} \\ 1\end{array}\right)$.

In the Dirac representation $\hat{h}_{D}$ can be regarded as the vector in Pauli algebra, with $\epsilon c p$, the energy due to the momentum, the component in the $\sigma^{1}$ axis, 0 the component in the $\sigma^{2}$ axis and $m c^{2}$, the energy due to the mass, the component in the $\sigma^{3}$ axis and finally the intensity of the vector is the energy $E=+\sqrt{c^{2} p^{2}+m^{2} c^{4}}$ of the particle.

The sign of $\operatorname{spin} \epsilon$ takes the values +1 for spin up and -1 for spin down.

In the following table we give some hamiltonians deduced from the Dirac equation in solving the equation by using the Kronecker product, with the corresponding representations [2], which are the six representations in [3].

Representation

$$
\begin{aligned}
& \sigma^{3} \otimes \sigma^{0}, i \sigma^{2} \otimes \sigma^{1}, i \sigma^{2} \otimes \sigma^{2}, i \sigma^{2} \otimes \sigma^{3} \text { (Dirac) } \\
& \sigma^{2} \otimes \sigma^{0},-i \sigma^{3} \otimes \sigma^{1},-i \sigma^{3} \otimes \sigma^{2},-i \sigma^{3} \otimes \sigma^{3} \\
& \sigma^{1} \otimes \sigma^{0}, i \sigma^{3} \otimes \sigma^{1}, i \sigma^{3} \otimes \sigma^{2}, i \sigma^{3} \otimes \sigma^{3} \\
& \sigma^{3} \otimes \sigma^{0},-i \sigma^{2} \otimes \sigma^{1},-i \sigma^{2} \otimes \sigma^{2},-i \sigma^{2} \otimes \sigma^{3} \\
& \sigma^{1} \otimes \sigma^{0},-i \sigma^{2} \otimes \sigma^{1},-i \sigma^{2} \otimes \sigma^{2},-i \sigma^{2} \otimes \sigma^{3} \text { (Weyl) } \\
& \sigma^{2} \otimes \sigma^{0}, i \sigma^{1} \otimes \sigma^{1}, i \sigma^{1} \otimes \sigma^{2}, i \sigma^{1} \otimes \sigma^{3}
\end{aligned}
$$

The Hamiltonian

$$
\begin{aligned}
& \epsilon c p \sigma^{1}+m c^{2} \sigma^{3} \\
& \epsilon c p \sigma^{1}+m c^{2} \sigma^{2} \\
& m c^{2} \sigma^{1}+\epsilon c p \sigma^{2} \\
& \epsilon c p \sigma^{2}+m c^{2} \sigma^{3} \\
& m c^{2} \sigma^{1}+\epsilon c p \sigma^{3} \\
& m c^{2} \sigma^{2}+\epsilon c p \sigma^{3}
\end{aligned}
$$

We would like to point out that for the more general case of the DiracSidharth equation [4],

$$
i \hbar \gamma^{\mu} \partial_{\mu} \psi-m c \psi-i \sqrt{\alpha} \ell \hbar \gamma_{D}^{5} \Delta \psi=0
$$

with $\gamma_{D}^{5}=i \gamma_{D}^{0} \gamma_{D}^{1} \gamma_{D}^{2} \gamma_{D}^{3}=\sigma^{1} \otimes \sigma^{0}, \Delta=\frac{\partial^{2}}{\partial x_{1}^{2}}+\frac{\partial^{2}}{\partial x_{2}^{2}}+\frac{\partial^{2}}{\partial x_{3}^{2}}, \alpha$ a dimensionless constant and $\ell$ is the physical length scale, the search for solutions in the form of plane waves yields, in the Dirac representation, the Hamiltonian

$$
\hat{h}_{D}=\epsilon c p \sigma^{1}-c \sqrt{\alpha} p^{2} \frac{\ell}{\hbar} \sigma^{2}+m c^{2} \sigma^{3}
$$

From now on let us use the natural unit $c=1$ and $\hbar=1$, and the Dirac representation. The reason of the choice of the Dirac representation will be justified later.

\section{Application to Two Flavor Neutrino Oscil- lation}

In this section, we are going to calculate the probability of the event $\left\{\nu_{\mu} \longrightarrow \nu_{\tau}, t\right\}$, for the transition from muon neutrino $\nu_{\mu}$ at time $t=0$ to tau neutrino $\nu_{\tau}$ 
at time $t$, in atmospheric neutrinos which is the simplest case of two flavor neutrino oscillations, observed by Fukuda et al [7]. We will take in consideration the positive energy and the negative energy mass eigenstates.

So, we should consider the following events:

$\left\{S i g E_{2}=S i g E_{3}\right\}$ : the two mass eigenstates have the same sign of energy at time $t=0$;

$\left\{\operatorname{Sig}_{2} \neq \operatorname{Sig}_{3}\right\}$ :the two mass eigenstates have the different signs of energy at time $t=0$.

The law of total probability and the multiplication theorem in probability calculus give us the transition probability

$$
\begin{aligned}
P\left(\nu_{\mu} \longrightarrow \nu_{\tau}, t\right)= & P\left\{\operatorname{SigE}_{2}=\operatorname{SigE}_{3}\right\} P\left(\nu_{\mu} \longrightarrow \nu_{\tau}, t / \operatorname{SigE}_{2}=\operatorname{SigE}_{3}\right) \\
& +P\left\{\operatorname{SigE}_{2} \neq \operatorname{SigE}_{3}\right\} P\left(\nu_{\mu} \longrightarrow \nu_{\tau}, t / \operatorname{Sig} E_{2} \neq \operatorname{Sig} E_{3}\right)
\end{aligned}
$$

where $P\left(\nu_{\mu} \longrightarrow \nu_{\tau}, t / S i g E_{2}=S i g E_{3}\right)$ is the probability of $\left\{\nu_{\mu} \longrightarrow \nu_{\tau}, t\right\}$ knowing that the two energy have the same sign at time $t=0$. This probability is equal to the Pontecorvo transition probability. For calculating this probability we can think of as a rotation in an another plane, as in the two-quark case (see, for example, [5])
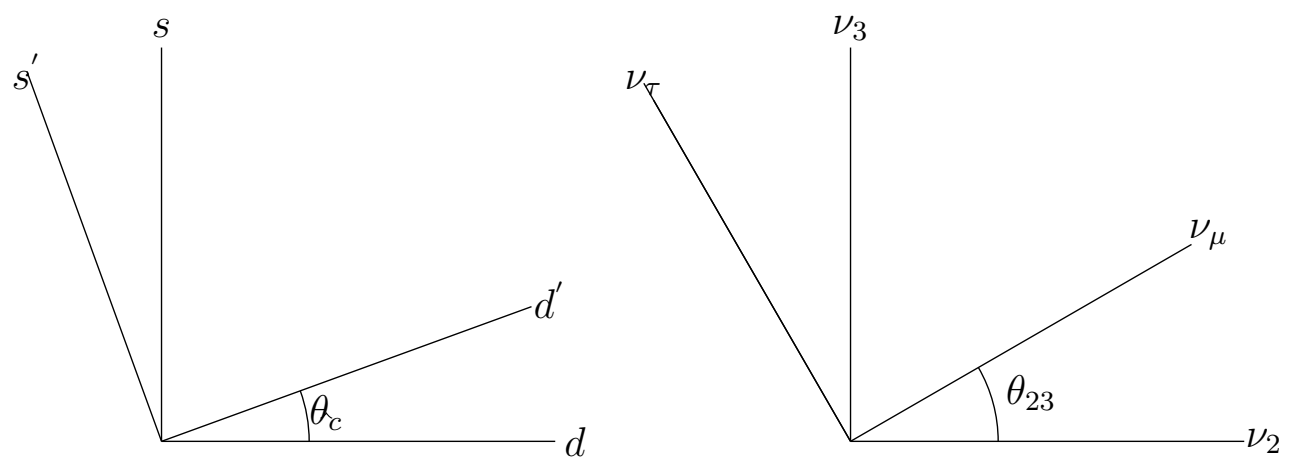

$$
\begin{aligned}
\left|\nu_{\mu}(t)\right\rangle & =\cos \theta_{23}\left|\nu_{2}(t)\right\rangle+\sin \theta_{23}\left|\nu_{3}(t)\right\rangle \\
\left|\nu_{\tau}(t)\right\rangle & =-\sin \theta_{23}\left|\nu_{2}(t)\right\rangle+\cos \theta_{23}\left|\nu_{3}(t)\right\rangle
\end{aligned}
$$

where $\left|\nu_{2}(t)\right\rangle=\left|\nu_{2}(0)\right\rangle e^{-i E_{2} t}$ and $\left|\nu_{3}(t)\right\rangle=\left|\nu_{3}(0)\right\rangle e^{-i E_{3} t}$.

$$
P\left(\nu_{\mu} \longrightarrow \nu_{\tau}, t / S i g E_{2}=S i g E_{3}\right)=\sin ^{2}\left(2 \theta_{23}\right) \sin ^{2}\left(\frac{E_{3} t-E_{2} t}{2}\right)
$$


with $E_{2}=\sqrt{p_{2}^{2}+m_{2}^{2}}$ and $E_{3}=\sqrt{p_{3}^{2}+m_{3}^{2}}$.

By using the same method

$$
P\left(\nu_{\mu} \longrightarrow \nu_{\tau}, t / S i g E_{2} \neq \operatorname{Sig} E_{3}\right)=\sin ^{2}\left(2 \theta_{23}\right) \sin ^{2}\left(\frac{E_{3} t+E_{2} t}{2}\right)
$$

Now, we just need to calculate the probabilities $P\left\{S i g E_{2}=S i g E_{3}\right\}$ and $P\left\{S i g E_{2} \neq S i g E_{3}\right\}$. For calculating them consider the angle $\theta$ between the two mass eigenstates with positive energies

$$
\begin{aligned}
& \nu_{2}(0)=\left|\nu_{2}(0)\right\rangle=\sqrt{\frac{E_{2}+m_{2}}{2 E_{2}}}\left(\begin{array}{c}
1 \\
\frac{\epsilon_{2} p_{2}}{E_{2}+m_{2}}
\end{array}\right) \\
& \nu_{3}(0)=\left|\nu_{3}(0)\right\rangle=\sqrt{\frac{E_{3}+m_{3}}{2 E_{3}}}\left(\begin{array}{c}
1 \\
\frac{\epsilon_{3} p_{3}}{E_{3}+m_{3}}
\end{array}\right)
\end{aligned}
$$

and between the two mass eigenstates with negative energies

$$
\begin{aligned}
& \overline{\nu_{2}}(0)=\left|\nu_{2}(0)\right\rangle=\sqrt{\frac{E_{2}+m_{2}}{2 E_{2}}}\left(\begin{array}{c}
-\frac{\epsilon_{2} p_{2}}{E_{2}+m_{2}} \\
1
\end{array}\right) \\
& \overline{\nu_{3}}(0)=\left|\nu_{3}(0)\right\rangle=\sqrt{\frac{E_{3}+m_{3}}{2 E_{3}}}\left(\begin{array}{c}
-\frac{\epsilon_{3} p_{3}}{E_{3}+m_{3}} \\
1
\end{array}\right)
\end{aligned}
$$

at time $t=0$.

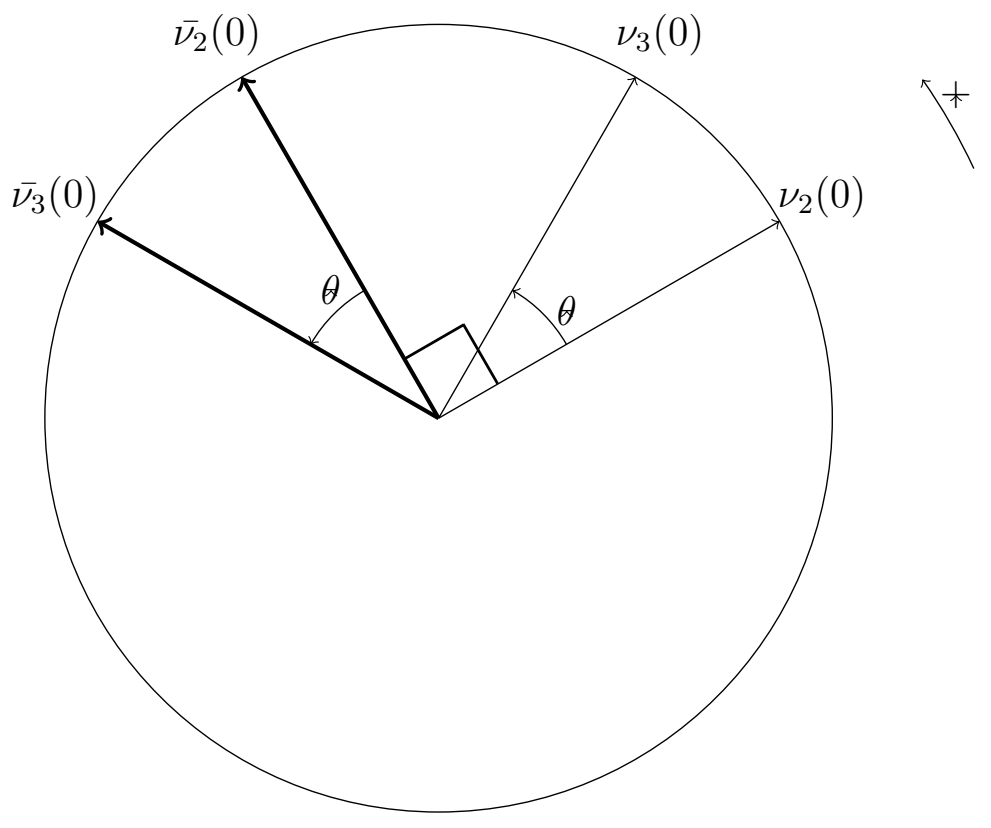




$$
\begin{gathered}
\cos \theta=\left\langle\nu_{2}(0) \mid \nu_{3}(0)\right\rangle=\sqrt{\frac{\left(E_{2}+m_{2}\right)\left(E_{3}+m_{3}\right)}{4 E_{2} E_{3}}}\left(1+\frac{\epsilon_{2} \epsilon_{3} p_{2} p_{3}}{\left(E_{2}+m_{2}\right)\left(E_{3}+m_{3}\right)}\right) \\
\sin \theta=\operatorname{det}\left(\left|\nu_{2}(0)\right\rangle,\left|\nu_{3}(0)\right\rangle\right)=\sqrt{\frac{\left(E_{2}+m_{2}\right)\left(E_{3}+m_{3}\right)}{4 E_{2} E_{3}}}\left(\frac{\epsilon_{3} p_{3}}{E_{3}+m_{3}}-\frac{\epsilon_{2} p_{2}}{E_{2}+m_{2}}\right) \\
\left|\nu_{3}(0)\right\rangle=\cos \theta\left|\nu_{2}(0)\right\rangle+\sin \theta\left|\overline{\nu_{2}}(0)\right\rangle \\
\left|\overline{\nu_{3}}(0)\right\rangle=-\sin \theta\left|\nu_{2}(0)\right\rangle+\cos \theta\left|\overline{\nu_{2}}(0)\right\rangle
\end{gathered}
$$

Again using the law of total probability and the multiplication theorem in probability calculus it follows the following probabilities

$$
P\left\{S i g E_{2}=S i g E_{3}\right\}=\cos ^{2} \theta \text { and } P\left\{S i g E_{2} \neq S i g E_{3}\right\}=\sin ^{2} \theta
$$

Hence

$P\left(\nu_{\mu} \longrightarrow \nu_{\tau}, t\right)=\sin ^{2}\left(2 \theta_{23}\right)\left(\cos ^{2} \theta \sin ^{2}\left(\frac{E_{3} t-E_{2} t}{2}\right)+\sin ^{2} \theta \sin ^{2}\left(\frac{E_{3} t+E_{2} t}{2}\right)\right)$

The same formula as obtained in [6]. In the case where, $\epsilon_{2}=\epsilon_{3}=1$ and $p_{2}=p_{3}=p$

$$
\begin{aligned}
& \cos \theta=\sqrt{\frac{\left(E_{2}+m_{2}\right)\left(E_{3}+m_{3}\right)}{4 E_{2} E_{3}}}\left(1+\frac{p^{2}}{\left(E_{2}+m_{2}\right)\left(E_{3}+m_{3}\right)}\right) \\
& \sin \theta=p \sqrt{\frac{\left(E_{2}+m_{2}\right)\left(E_{3}+m_{3}\right)}{4 E_{2} E_{3}}}\left(\frac{1}{E_{3}+m_{3}}-\frac{1}{E_{2}+m_{2}}\right)
\end{aligned}
$$

and in the relativistic limit $\cos ^{2} \theta \longrightarrow 1, \sin ^{2} \theta \longrightarrow 0[6,8]$. Thus the Pontecorvo transition probability is recovered in the relativistic limit.

\section{Conclusion}

The hamiltonian is an operator whose eigenvalues are the positive energy and negative energy in the Dirac theory of a free spin- $\frac{1}{2}$ fermion. It is a vector in Pauli algebra, that is a linear combination of the Pauli matrices. The coefficients of the linear combination are the different forms of the energy. So, it is a candidate for replacing the energy approximation which lead to 
the Pontecorvo neutrino oscillation formulas.

We have obtained the same formula as obtained by [6]. We can see that the spins at time $t=0$ represented by their signs $\epsilon_{2}$ and $\epsilon_{3}$ have impact on the transition probability. For $\epsilon_{2}=\epsilon_{3}=1, p_{2}=p_{3}=p$, in the relativistic limit the Pontecorvo transition probability is recovered.

\section{References}

[1] Raoelina Andriambololona, Rakotonirina C., A Study of the DiracSidharth Equation, EJTP 8, No.25, 177-182, 2011.

[2] Rakotonirina C., Produit Tensoriel de Matrices en Théorie de Dirac, Thèse de Doctorat de Troisième Cycle, Université d'Antananarivo, Antananarivo, Madagascar, pp 62-63, 2003.

[3] Wang R.P., Varieties of Dirac Equation and Flavors of Leptons and Quarks, arXiv:hep-ph/0107184.

[4] Sidharth, B.G., The Modified Dirac Equation, EJTP 7, No.24, 211-218, 2010 .

[5] Bettini A., Introduction to Elementary Particle Physics, Cambridge University Press, 358-360, 2008.

[6] Blasone M., P A. Henning P. A., Vitiello G., The exact formula for neutrino oscillations, Phys.Lett. B451 (1999) 140-145. arXiv:hepth/9803157.

[7] Super-Kamiokande Collaboration: Fukuda Y. et al, Evidence for oscillation of atmospheric neutrinos, Phys.Rev.Lett.81:1562-1567,1998. arXiv:hep-ex/9807003.

[8] Ohlsson T. Dynamics of Quarks and Leptons: Theoretical Studies of Baryons and Neutrinos, Scientific thesis for the degree of Doctor of Philosophy (PhD), KTH Högskoletryckeriet, Stockholm 2000, 98. 